\title{
Interpenetrating Polymer Network Hydrogel Membranes of Karayagum and Sodium Alginate for Control Release of Flutamide Drug
}

\author{
Guruguntla Nagarjuna ${ }^{1}$, Palla Kumara Babu ${ }^{1}$, Yeggada Maruthi ${ }^{1}$, Areti Parandhama ${ }^{1}$, Chintha Madhavi ${ }^{1}$, \\ M. C. S. Subha ${ }^{2}$, Kashayi Chowdojirao ${ }^{1 *}$ \\ ${ }^{1}$ Department of Polymer Science \& Technology, Sri Krishnadevaraya University, Anantapur, India. \\ ${ }^{2}$ Department of Chemistry, Sri Krishnadevaraya University, Anantapur, India.
}

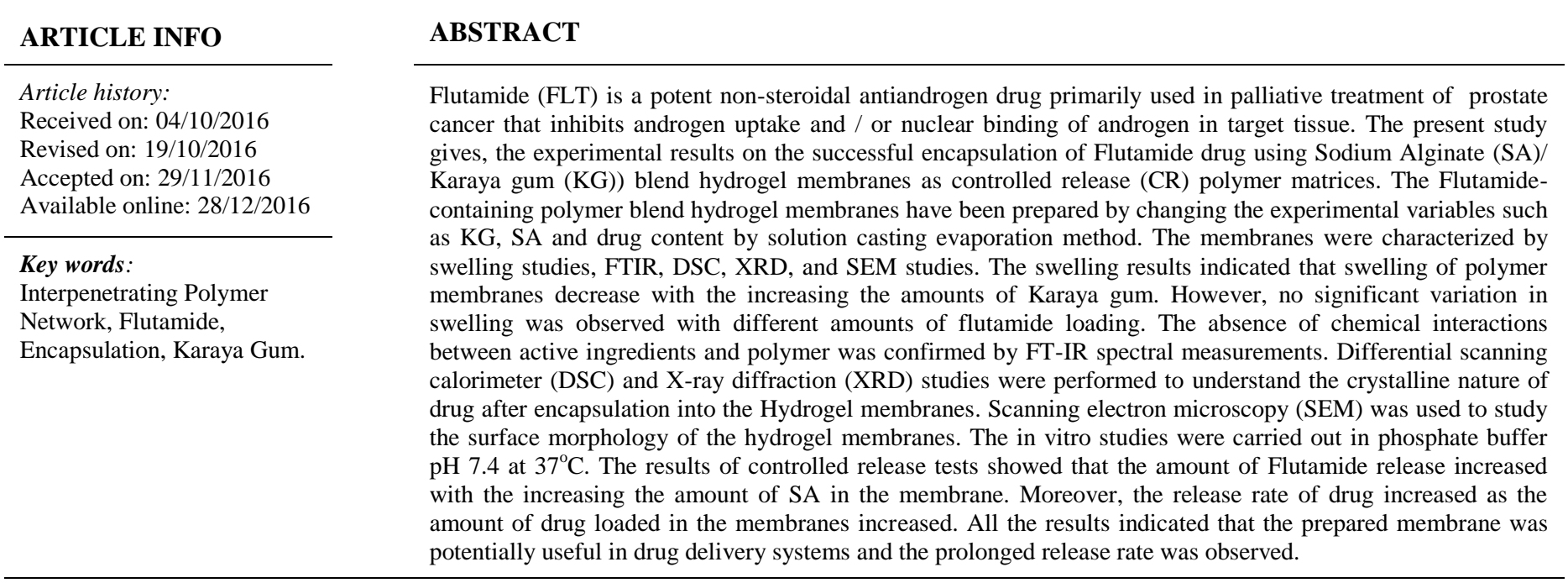

\section{INTRODUCTION}

In recent years, biodegradable polymers have attracted attention to be used as biomaterials particularly, for tissue engineering, gene therapy, wound healing and controlled drug delivery systems (Sahoo et al., 2009). The most important advantage of biodegradable polymers is the disappearance of implanted foreign materials from the body as a result of their biodegradation. In general polymers used in biomedical

* Corresponding Author
E-mail: chowdojirao @ gmail.com applications are poly (lactic acid), poly(glycolic acid) (PGA), poly(e-caprolactone) (PCL), poly(3-hydroxyl butyrate) (PHB), copolymers of polyglycolide, chitosan, alginate and natural gums such as Xanthan Gum, Guar gum (Sahoo et al., 2010; Edulund et al., 2002; Jain Rajeev et al., 2002; Kamath et al., 1993).

Different types of drug delivery systems, like microcapsules, microspheres, implants, pallets and hydrogel membranes have been fabricated by using above bio-degradable polymers as drug matrix. Hydrogel membranes play a major role in medical applications, in particular in a number of life saving treatment methods. Membranes are used in drug delivery, artificial organs, tissue regeneration, diagnostic devices, as coatings for medical devices, bio-separations, etc. 
The total membrane area produced for medical applications almost matches all industrial membrane applications together (Fortunati et al., 2012; Okuno et al., 1992; Zhang et al., 1997; Chanachi et al., 2000; Cao et al., 2000). In fact in true sense, the value of medical membrane products is far larger than all other applications combined (Soppimath et al., 2001; Gardner et al., 1983; Gregoriadis et al., 1977; Gregoriadis et al., 2007). The biggest part of the medical market involves membranes in drug delivery, hemo-dialysis, other artificial organs (oxygenators, pancreas, etc.) and tissue engineering. Karaya Gum is a complex polysaccharide. The primary structure is shown to be composed of D-glucuronic acid, D-galacturonic acid, D-galactose, L-rhamnose and acetyl groups, in different proportions according to quality, type, and origin of the polysaccharide (Yuan et al., 2007). The average composition of acetyl free samples is $60 \%$ of neutral sugar (rhamnose and galactose) and $40 \%$ of acidic sugar residues (galacturonic acid and glucuronic acid) (Du et al., 2006).Native polysaccharide contains approximately $8 \%$ of acetyl groups (Paula et al., 2002).

As Karaya gum is a natural polymer and biodegradable one it is used for many applications, with its properties such as high degree of transparency and biocompatibility and high melting point $\left(185.6^{\circ} \mathrm{C}\right)$. Currently, there is an increasing interest in using Karaya Gum for biodegradable materials even in biomedical applications because of its biocompatibility. As there were no reports on the drug delivery applications of Karaya Gum / SA blends the author thought to work on this system. To tailor its crystallization behaviour, degradation rate and other material properties for the applications, blending Karaya Gum with other biocompatible polymers like sodium alginate, it is practically and economically new approach in drug delivery studies.

Among the biocompatible and natural polymers, sodium alginate (SA) is a water-soluble polysaccharide having good membrane forming properties (Mochizuki et al., 1977). Alginate is a linear chain structure of $(1 \rightarrow 4)$-linked $\beta$-D-mannuronic acid $(\mathrm{M})$ and $\alpha$-L-guluronic $\operatorname{acid}(\mathrm{G})$ arranged in block wise fashion as shown below. These blocks are constructed in three different ways: homopolymeric MM blocks, homopolymeric GG blocks and heteropolymeric sequentially alternating MG blocks (Fischer et al., 1955; Haung et al., 1966). Presence of $\alpha$-L-guluronic acid in various ratios and molecular weight alters physico-chemical properties of the polymer (Moe et al., 1995). These factors contribute towards the overall drug delivery performance of the membrane.

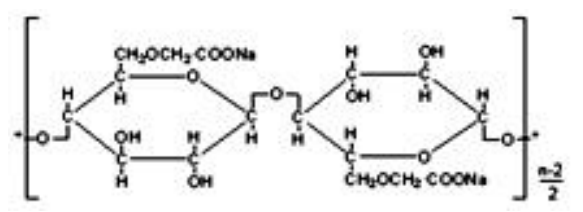

Chemical structure Sodium alginate

Flutamide is a synthetic, non-steroidal, pure antiandrogen used primarily to treat prostate cancer (Sahoo et al., 2009). It acts as a silent antagonist of the androgen receptor (AR), competing with androgens such as testosterone and its powerful active metabolite dihydrotestosterone (DHT) for binding to ARs in the prostate gland. By doing so, it prevents them from stimulating the prostate cancer cells to grow. In addition to its use in prostate cancer, flutamide has been used to treat hyperandrogenism (excess androgen levels) in women, such as in those with polycystic ovary syndrome (PCOS), and hirsutism (Sahoo et al., 2010). Flutamide has been largely replaced by newer non-steroidal antiandrogens, namely bicalutamide, due to better safety, tolerability, and pharmacokinetic profiles (Edulund et al., 2002; Jain Rajeev et al., 2002). The Chemical structure of Flutamide is shown below.<smiles>CC(C)C(=O)Nc1ccc([N+](=O)[O-])c(C(F)(F)F)c1</smiles>

Chemical structure of Flutamide

In continuation of our drug delivery studies (Prabhakar et al., 2013 and C Venkata Prasad et al., 2012), we have prepared $\mathrm{SA} /$ Karaya gum hydrogel blend membranes for controlled release of Flutamide. It also deals with the in-vitro release studies on membrane formulations loaded with different amounts of Flutamide. Drug loaded membranes were characterized by using Fourier transform infrared spectroscopy (FT-IR), X-ray diffraction (X-RD), diffraction scanning calorimetry (DSC). The kinetics of the drug delivery system has been reported.

\section{EXPERIMENTAL}

\section{Materials}

SA of medium viscosity grade (viscosity of $1 \% \mathrm{w} / \mathrm{v}$ solution at $25{ }^{\circ} \mathrm{C}, 5.5 \pm 2$ counts per second) was obtained from $\mathrm{Hi}$ media, Hyderabad, India. Karaya Gum $(\mathrm{KG})$ and Flutamide were purchased from Aldrich chemicals (St. Louis) USA. Doubledistilled water was used throughout the research work.

\section{Preparation of drug loaded blend membrane}

The membranes of SA/ KG were prepared by a solventcasting method. $2 \%$ aqueous solution of SA was prepared by dissolving SA in water overnight under constant stirring conditions, to this the required amount of Flutamide drug was added slowly until a clear homogeneous solution is obtained. Karaya Gum solution is prepared separately by dissolving 100-200 $\mathrm{mg}$ of Karaya Gum in $20 \mathrm{ml}$ of water and pouring it into the drug (0.1, 0.2 and $0.3 \mathrm{gm}$ ) containing different SA solutions (as shown in Table 1) at room temperature under magnetic stirring for 15 min. The final solution obtained was used to prepare films by casting on a glass plate, followed by drying at $30{ }^{\circ} \mathrm{C}$ for $72 \mathrm{~h}$ and films with thickness $100-200 \mu \mathrm{m}$ were obtained. 


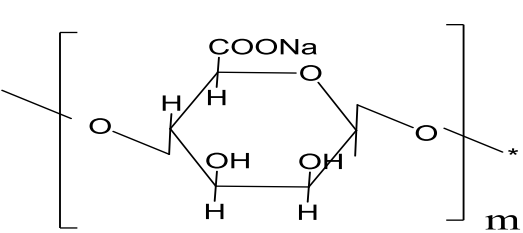

Sodium Alginate

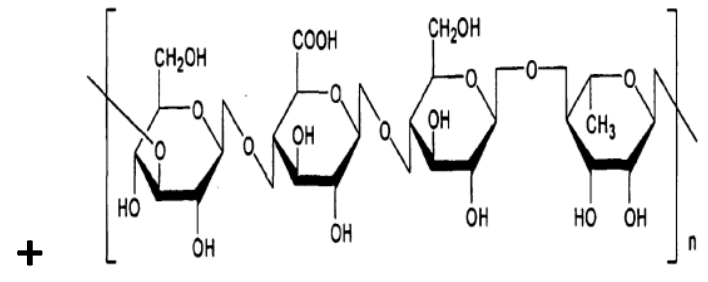

Karaya Gum
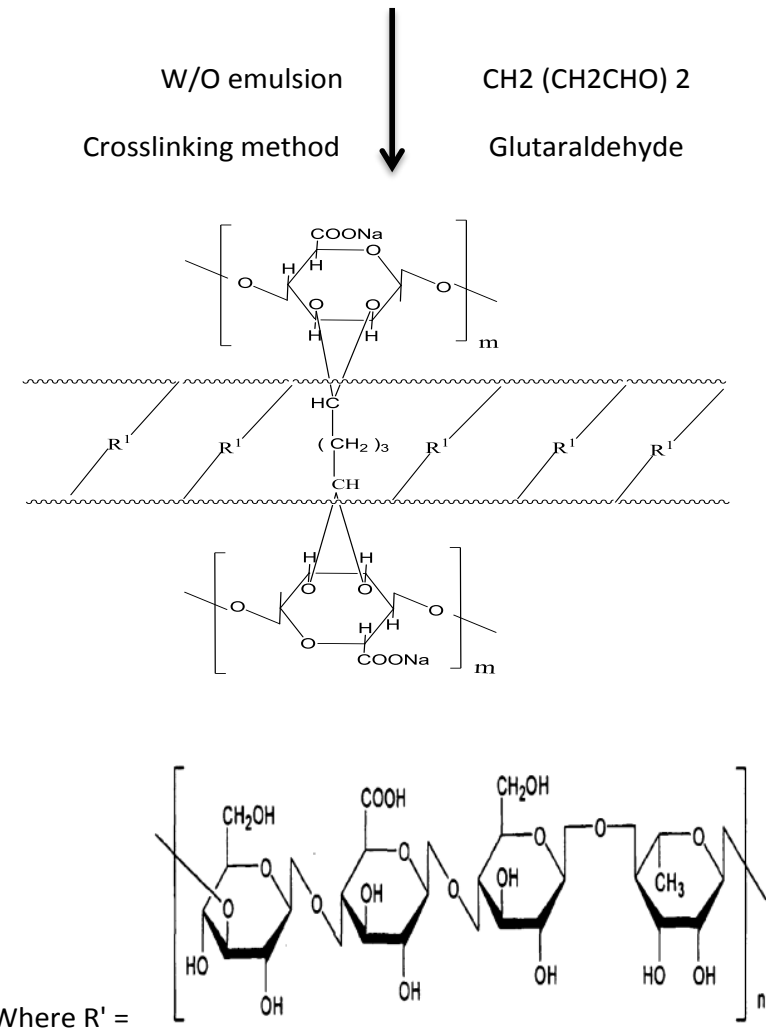

Scheme 1. Schematic representation of preparation of the cross linked SA and KG blend hydrogel membranes.

The dried membranes were dipped into acetone -water mixture containing different ratios (Table 1.) of GA and $0.1 \mathrm{HCl}$ for crosslinking upto $30 \mathrm{~min}$. Then the crosslinked membranes were washed with distilled water to remove the excess of unreacted GA and dried at $37^{\circ} \mathrm{C}$ for $24 \mathrm{~h}$. The schematic diagram of the formation of cross-linked blend membrane is shown in Scheme 1. The prepared membranes were stored in a closed container for further characterization. The formulation details are given in Table 1.

\section{Swelling properties}

The blend membrane samples of the size $1.5 \mathrm{~cm}^{2}$ squares were cut and dried at $60{ }^{\circ} \mathrm{C}$ until constant weight (Wd) was obtained. Then, they were immersed into $5 \mathrm{ml}$ of distilled water at $37{ }^{\circ} \mathrm{C}$ in 7.4 phosphate buffer solution for at least 24 hours. Afterwards, the samples were taken out from bottles and wiped with blotting paper to remove the surface adhered water molecules and weighed $\left(W_{s}\right)$. Here, the degree of swelling ratio $(D S)$ is defined as the weight of water absorbed by the membrane $\left(W_{s}\right)$ divided by the dry weight of the membrane $(\mathrm{Wd})$ as per equation (1).

$$
\% D S=\frac{(W s-W d)}{W_{d}} \times 100 \ldots . . \text { Equation } 1
$$

\section{Estimation of drug and encapsulation efficiency}

Polymer blend membranes equivalent to $100 \mathrm{mg}$ was stirred in $20 \mathrm{ml}$ of phosphate buffer solution ( $\mathrm{pH} 7.4)$ and the drug content was analyzed by UV spectrophotometer (Lab India, Mumbai, India) at a $\lambda_{\max }$ of $200 \mathrm{~nm}$. \% of Encapsulation efficiency (EE) was calculated as the percentage $(\mathrm{w} / \mathrm{w})$ of the theoretical drug content (Equation 2). Results were based on triplicate and the average values are compiled in Table 2 .

$$
\% \mathrm{EE}=\frac{\text { Actual drug loading }}{\text { Theoretical drug loading }} \mathrm{X} 100 \ldots \ldots \text { Equation } 2
$$




\section{In vitro release studies}

The drug loaded blend membranes were suspended in baskets containing $500 \mathrm{ml}$ of phosphate buffer solution (pH-7.4, acts as simulated intestinal fluid) and incubated on a shaking bed (Lab India, Mumbai, India) at $37{ }^{\circ} \mathrm{C}$ with a rotating speed of 100 rpm. At appropriate time intervals aliquot of the samples were withdrawn and the amount of Flutamide released from the membranes were evaluated by UV spectrophotometer (Lab India, Mumbai, India) at a $\lambda_{\max }$ of $200 \mathrm{~nm}$. Then an equal volume of the same dissolution medium was added back to maintain a constant volume. All three experiments were done in triplicate and results are averaged.

\section{Fourier transform infrared spectroscopy (FTIR)}

The FT-IR spectra were recorded on a FT-IR Spectrophotometer (Bomem, Model: MB3000, Canada). About $2 \mathrm{mg}$ of the sample was grounded thoroughly with $\mathrm{KBr}$ and pellets were made by using hydraulic press under a pressure of $600 \mathrm{~kg} / \mathrm{cm}^{2}$. Spectra were scanned between 4000 and $500 \mathrm{~cm}^{-1}$ at ambient temperature.

\section{Scanning Electron Microscopy (SEM)}

SEM images of drug loaded and unloaded SA/KG blend membranes in dry powder form were recorded using a JSM 6400 SEM (JEOL Ltd., Akishima, Tokyo, Japan) at $100 \times$ and $80 \times$ magnification. Working distance of $39 \mathrm{~mm}$ was maintained and the acceleration voltage used was $20 \mathrm{kV}$, with the secondary electron image (SEM) as a detector.

\section{Differential Scanning Calorimetry (DSC)}

The sample membranes were heated from 0 to $400{ }^{\circ} \mathrm{C}$ at a heating rate of $10{ }^{\circ} \mathrm{C} / \mathrm{min}$ under nitrogen atmosphere (flow rate, $20 \mathrm{ml} / \mathrm{min}$ ) using a DSC (Model-SDT Q600, USA) and then thermograms were obtained.

\section{X-Ray diffraction (X-RD)}

X-Ray diffraction patterns of Flutamide, SA/KG blend membrane and Flutamide -loaded SA/KG blend membranes were recorded using Shimadzu Lab-XRD-6000X diffractometer [Japan], using Nickel-filtered $\mathrm{Cu} \mathrm{K} \alpha$ radiation $[\lambda=0.154 \mathrm{~nm}]$. Dried membrane sample was mounted on a sample holder and the patterns were recorded in the range of $10-50^{\circ}$ at the speed of $5 \%$ min to know the crystallinity.

\section{RESULTS \& DISCUSSION}

\section{FTIR spectral analysis}

FT-IR spectra of drug (Flutamide) (a), Plain SA/KG blend membrane (b) and Drug loaded SA/KG membrane (c) are presented in Fig.1. FTIR studies were carried out to confirm the crosslinking between SA and KG membrane by GA. From Fig.1 (a) the FTIR spectra of Flutamide (a) indicates that the characteristic peaks of $-\mathrm{NO}, \mathrm{NH}, \mathrm{C}=\mathrm{O}$ and $-\mathrm{CH}_{2}$ are observed at $1345 \mathrm{~cm}^{-1}, 3390 \mathrm{~cm}^{-1}, 1721 \mathrm{~cm}^{-1}$ and $3111 \mathrm{~cm}^{-1}$ respectively. From Fig.1 (b) it is noticed that a characteristic peak of $3500 \mathrm{~cm}^{-1}$ indicates the presence of $-\mathrm{OH}$ group in $\mathrm{KG}$ and $1731 \mathrm{~cm}^{-1}$ and $1254 \mathrm{~cm}^{-1}$ indicates the presence of symmetric and symmetric stretching vibrations of acetyl groups of $\mathrm{KG}$ in blend membrane. Again the characteristics peaks at $1616 \mathrm{~cm}^{-1}$ and $1425 \mathrm{~cm}^{-1}$ noticed due to carboxylate group of the uronic acid residues in SA

Fig.1 (c) shows the FTIR spectra of SA/KG blend membrane cross linked with GA, the crosslinker. It can be noticed that band at 1616, 3473, 2900 and $1120 \mathrm{~cm}^{-1}$ are nearly associated with SA and also the presence of peaks related to carboxylic group and acetals form by cross linker reaction through glutaraldehyde with the alcohol groups of SA and acid groups from KG. These blends confirm the crosslinking between chains of KG \& SA by glutaraldehyde. From Fig.1 (c) it is also noticed that the flutamide peaks are not noticed which indicates the drug is uniformely dispersed in the blend membrane.

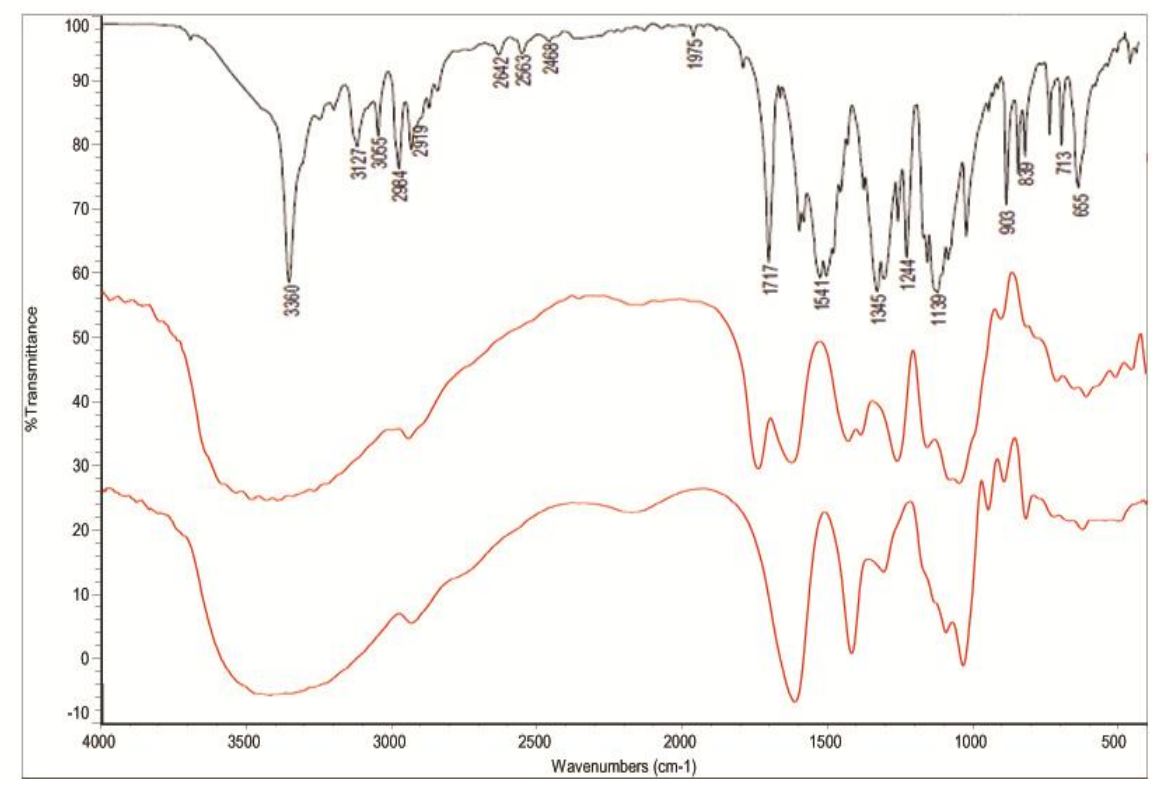

Fig. 1: FT-IR spectra of Flutamide (a), Plain SA/KARAYA GUM blend membrane (b) and Drug loaded SA/KARAYA GUM blend membrane (c). 


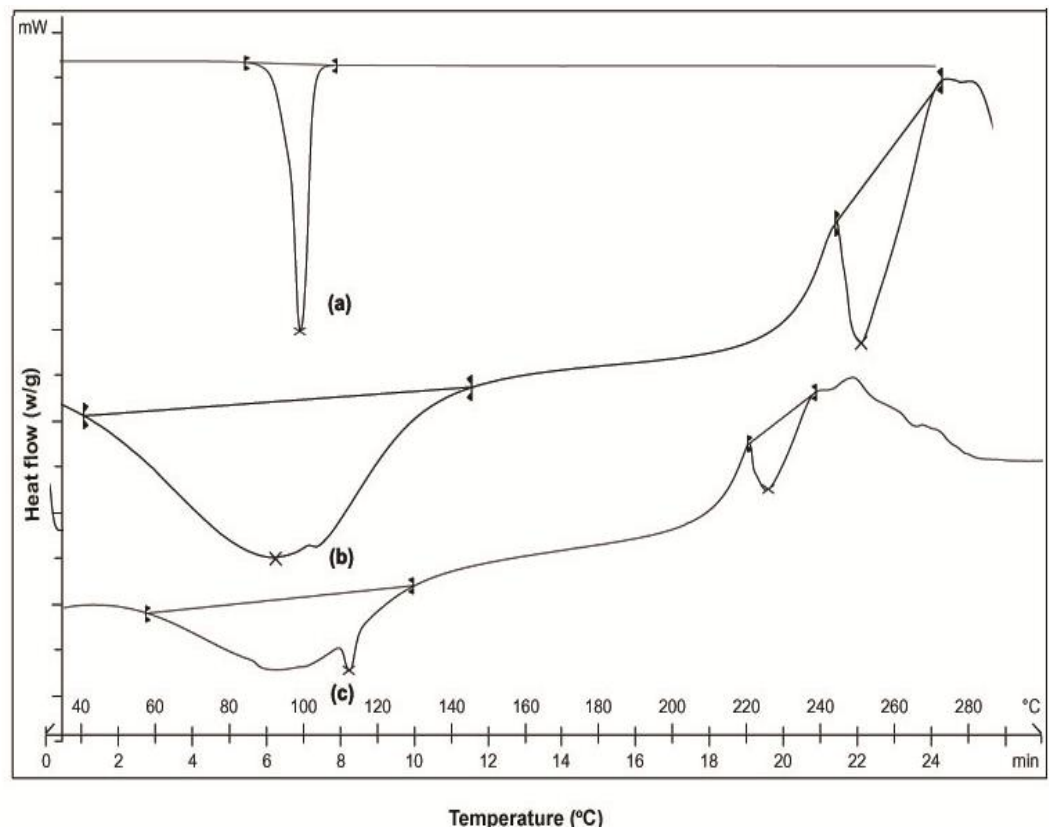

Fig. 2: DSC thermogramme of plain Flutamide drug (a), plain membrane (b) and drug loaded membrane (c).

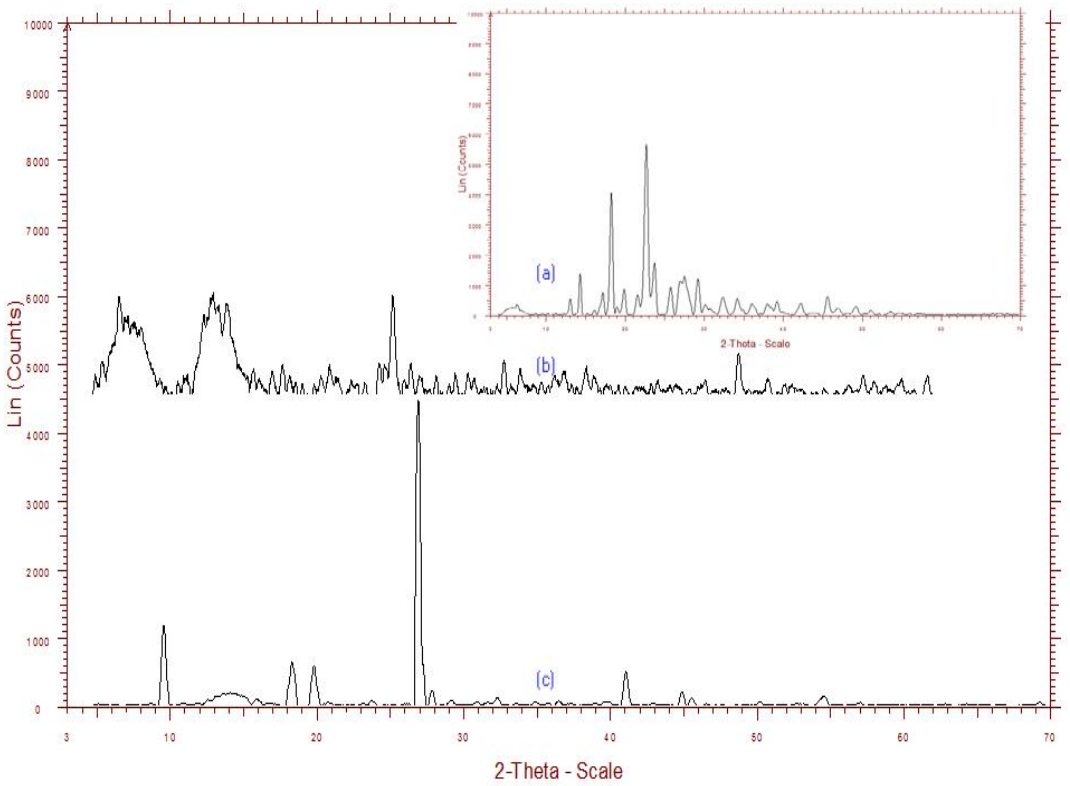

Fig. 3: X-Ray diffraction patterns of pure Flutamide drug (a), Plain membrane (b) and drug loaded SA/KG blend membrane (c).

\section{DSC Studies}

DSC thermograms of plain Flutamide drug (a), plain membrane (b) and drug loaded membrane(c) are presented in Fig. 2. In case of plain drug Fig. 2 (a) shows the peak at $95^{\circ} \mathrm{C}$ indicating the melting peak. From Fig.2 (b), it is noticed that the peak one at $90^{\circ} \mathrm{C}$ indicating $\mathrm{Tg}$ of the pure blend and other at $110^{\circ} \mathrm{C}$ indicating the melting point of the pure blend membrane. From Fig.2(c) it is observed that there are two broad peaks one at around $90^{\circ} \mathrm{C}$ which indicates the $\mathrm{Tg}$ value and other observed at $115^{\circ} \mathrm{C}$ indicates the melting temperature of the drug loaded blend membranes. The sharp peak of drug is not noticed either in pure blend membrane or in drug loaded blend membrane indicating the uniform distribution in the blend matrix.

\section{X-Ray diffraction (X-RD Ananlysis)}

X-RD diffractograms of plain Flutamide drug (a) Plain blend membrane (b) and Flutamide loaded SA/KG blend membrane (c) are presented in Fig.3. From Fig. 3 it is noticed that the plain drug has shown characteristic peaks at $2 \Theta=0$ to $20^{\circ}$ due to its crystalline nature, whereas the absence of these peaks of drug in drug either in plain membrane or in drug loaded membrane indicates the uniform distribution in the blend membrane matrix. 


\section{Scanning Electron Microscopy (SEM) Ananlysis}

The membrane obtained from SA/KG blend solution by conventional solvent casting method were morphologically studied with the help of SEM and these are shown in Fig. 4. The images of Fig. 4 (a) \& 4 (b) shows SA/KG (90/10) membrane cross-linked with $0.1 \% \mathrm{GA}$ without drug content at different resolutions. The photographs 4(a) \& 4(b) indicates the complete miscibility of these polymers in the blend. Fig 4(c) \& 4 (d) shows SA/KG (90/10) blend membrane with drug and cross-linked with $0.1 \%$ GA at different resolutions. From these photographs Fig. 4 (c \& d) the drug molecules are physically associated and adsorbed at the surface of the polymer membrane. This clearly indicates good compatibility between the polymer matrix and Flutamide drug.
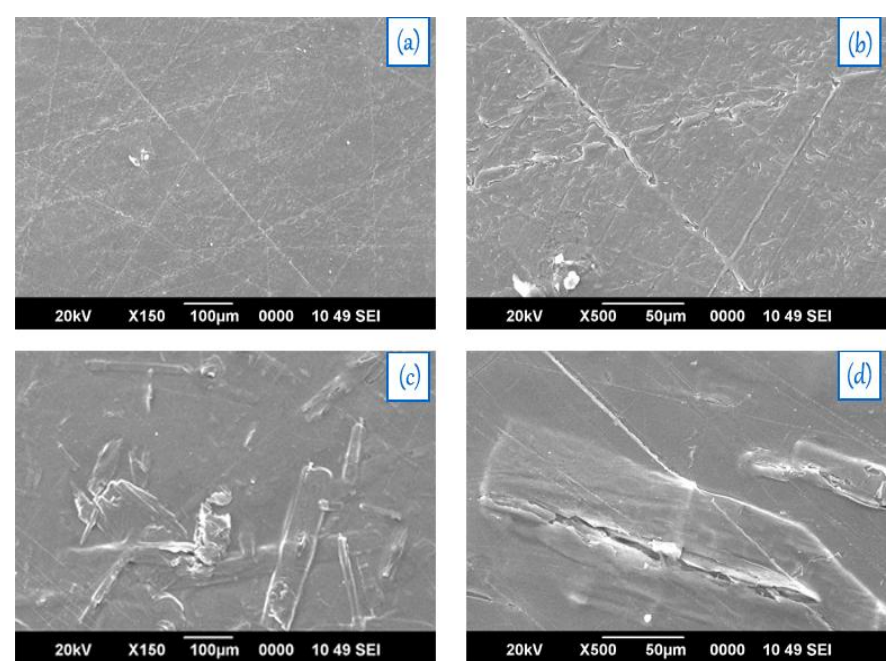

Fig. 4: SEM photographs of (a) \& (b) plain membranes (SKF-8) and (c) \& (d) drug-loaded membranes (SKF-4) at different resolutions.

\section{Swelling properties}

The ability of a blend membrane to preserve water is an important aspect to be investigated for drug delivery applications. The water uptake (swelling) percent of blend membrane with different compositions of SA, KG and GA in the blend membranes are shown in Fig. 5 ( $\mathrm{a} \& \mathrm{~b}$ ).

As seen here Fig. 5(b), the blend membranes did absorb water rather fast in about $4 \mathrm{~h}$, and then gradually reached equilibrium in beyond this time. The water binding ability of the blend membrane could be mainly attributed to the hydrophilic nature of both SA and $\mathrm{KG}$ present in the blend membrane (Fig.5.a). In general, the water uptake decreases as the crosslinking degree is increased because of the decrease in the number of hydrophilic groups as well as the more difficulty in the structural expansion due to the more dense covalently linked network (Prabhakar et al., 2013), which is also evident from the Fig. 5 (a) in the present study.

For the specific case of Sodium Alginate, a more complex relationship is found between cross-linking degree with Glutaraldehyde and swelling capability, because the crystalline content in the material is also changing (Venkata prasad et al., 2012). The results in Fig. 5 (a) indicate that the percent of swelling increases with increasing KG composition in the blend membrane.

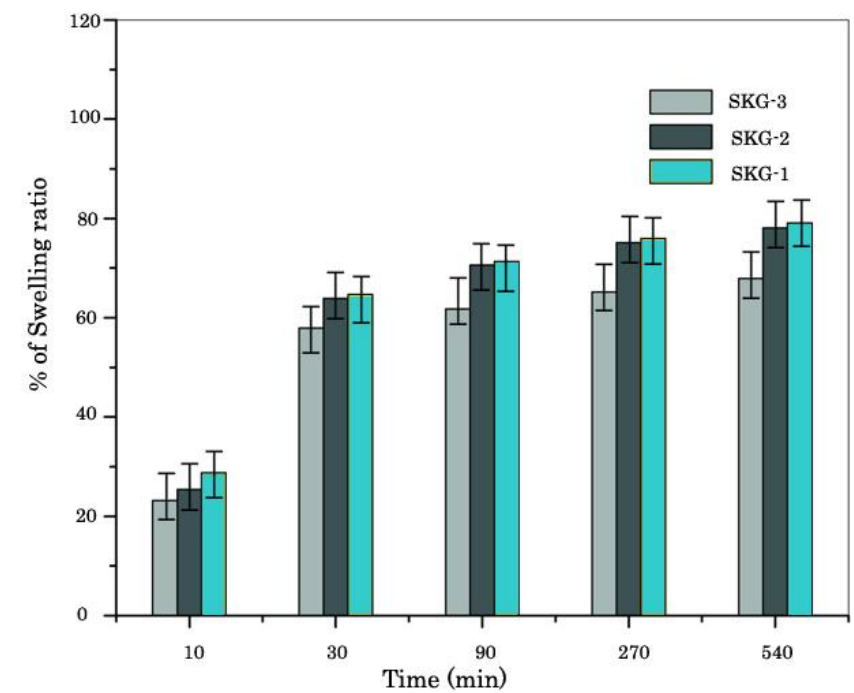

Fig. 5 (a): Variation of \% swelling ratio with time for of different compositions of (Sodium alginate / Karaya gum) blend membranes

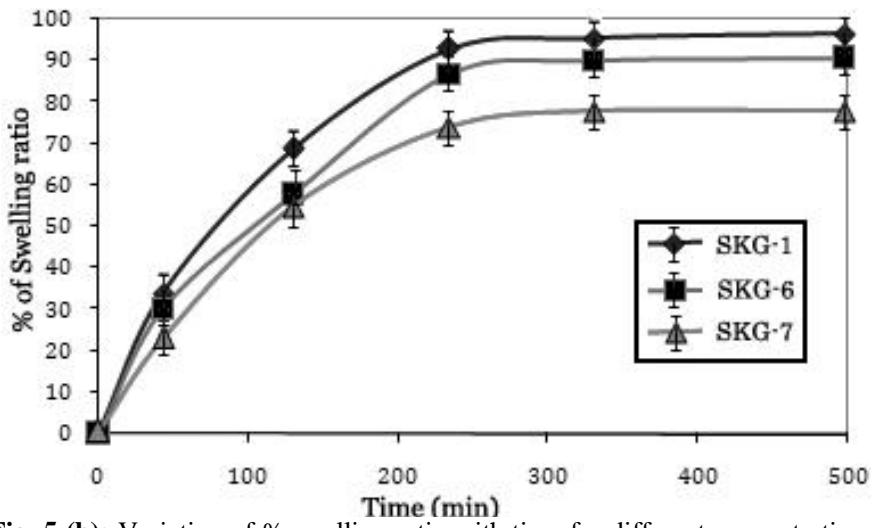

Fig. 5 (b): Variation of \% swelling ratio with time for different concentrations of the crosslinker (GA) in different SA / KG blend membranes

\section{Encapsulation efficiency}

Three different concentrations of Flutamide (0.1, 0.2 and $0.3 \mathrm{gm}$ ) were loaded during the preparation of blend membranes. Results of \% encapsulation efficiency are included in Table 1. The results indicate that the percentage of encapsulation efficiency depends on the preparation conditions and the type of the matrix material used. Table. 1, shows that the $\%$ of encapsulation efficiency increased with increasing amount of drug loaded in the range of 63 to $75 \%$. Encapsulation efficiency decreased with increase in cross-linker (GA) content, this decrease might be due to the increase in crosslinking of the polymer matrix in the range of 70.0 to 63.0 (Table 1). The increase in cross-linking density leads to the formation of rigid structure as a result, reduction in free volume within the polymer matrix, thereby reducing their encapsulation efficiencies. The encapsulation efficiency decreased 
with in the amount of $\mathrm{KG}$ in polymer blend membrane for compositions SKG-1, SKG-2 \& SKG -3 from 69.8 to 62.5 .

Table 1: Polymer blend composition at various concentrations of reaction mixture components and their encapsulation efficiency.

\begin{tabular}{cccccc}
\hline $\begin{array}{c}\text { Sample } \\
\text { code }\end{array}$ & $\begin{array}{c}\text { SA } \\
(\mathbf{w} / \mathbf{w})\end{array}$ & $\begin{array}{c}\text { KG } \\
(\mathbf{w} / \mathbf{w})\end{array}$ & $\begin{array}{c}\text { GA } \\
(\boldsymbol{\%})\end{array}$ & $\begin{array}{c}\text { Drug } \\
(\mathbf{g m})\end{array}$ & $\begin{array}{c}\text { \% Encapsulation } \\
\text { efficiency }\end{array}$ \\
\hline SKG-0 & 0.9 & 0.1 & 0.01 & - & - \\
SKG-1 & 0.9 & $\mathbf{0 . 1}$ & $\mathbf{0 . 0 1}$ & 0.1 & $69.8 \pm 1.5$ \\
SKG-2 & 0.85 & $\mathbf{0 . 1 5}$ & 0.01 & 0.1 & $65.2 \pm 1.1$ \\
SKG-3 & 0.8 & $\mathbf{0 . 2}$ & 0.01 & $\mathbf{0 . 1}$ & $\mathbf{6 2 . 5} \pm \mathbf{0 . 8}$ \\
SKG-4 & 0.9 & 0.1 & 0.01 & $\mathbf{0 . 2}$ & $\mathbf{7 0 . 8} \pm \mathbf{1 . 2}$ \\
SKG-5 & 0.9 & 0.1 & 0.01 & $\mathbf{0 . 3}$ & $\mathbf{7 4 . 2} \pm \mathbf{0 . 9}$ \\
SKG-6 & 0.9 & 0.1 & $\mathbf{0 . 0 5}$ & 0.1 & $64.6 \pm 1.3$ \\
SKG-7 & 0.9 & 0.1 & $\mathbf{0 . 1}$ & 0.1 & $62.4 \pm 0.5$ \\
\hline
\end{tabular}

\section{In-vitro release study}

In-vitro release studies were performed by dissolution test apparatus in $\mathrm{pH} 7.4$ buffer solutions, and the results are discussed below.

\section{Effect of Polymers}

To understand the release profiles of Flutamide from cross-linked $\mathrm{SA} / \mathrm{KG}$ blend membranes, in-vitro release studies were carried out in $\mathrm{pH} 7.4$ phosphate buffer solutions at $37^{\circ} \mathrm{C}$ and are shown in Fig. 6 (a, b \& c). Fig 6 (a) shows the cumulative release of Flutamide through membranes containing different ratios of SA/KG at constant GA $(0.1 \%)$ and at constant drug $(0.1)$ concentration. From Fig. 6 (a) it was observed that the highest cumulative release of drug is obtained for $\mathrm{SA} / \mathrm{KG}$ formulation $(0.9 \mathrm{SA} / 0.1 \mathrm{KG})$. On the other hand, the least cumulative release of drug is obtained for $\mathrm{SA} / \mathrm{KG}$ formulation $(0.8 \mathrm{SA} / 0.2 \mathrm{KG})$. When the amount of Karaya gum is increased in the $\mathrm{SA} / \mathrm{KG}$ blend membrane, a decrease in the drug release observed.

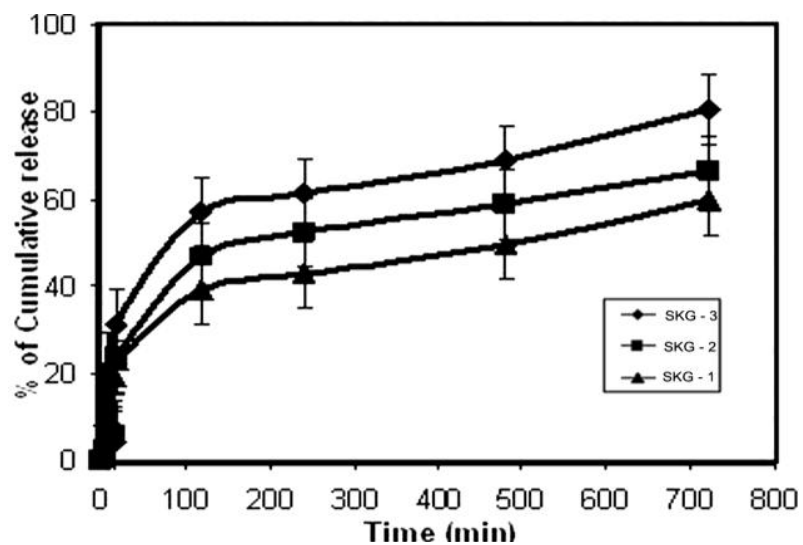

Fig. 6 (a): Percentage cumulative release of Flutamide through SA/KG blend hydrogel membranes containing different amounts of KG: $(\downarrow) 20 \% \mathrm{KG}$, (घ) $15 \% \mathrm{KG}$, and $(\boldsymbol{\Delta}) 10 \% \mathrm{KG}$ at $\mathrm{pH} 7.4$

This could be because of the increase of $\mathrm{KG}$ content in the blend membrane, where the hydrophobicity of the overall matrix increases due to the presence of ester linkages and methyl groups of Karaya gum, which further increases the hydrophobicity of the matrix, thereby decreasing the release rates of drug. This will further decrease the molecular volume of the hydrated polymer due to decreased swelling of Karaya gum component of the polymer matrix, thereby reducing the free volumes spaces of the matrix. Hence, with an increase of Karaya gum in the SA/KG blend membranes a decrease in $\%$ of cumulative release is observed.

\section{Effect of drug concentration}

Fig. 6 (b) shows the cumulative drug release particles from the $\mathrm{SA} / \mathrm{KG}$ blend membranes loaded with different amounts of drug $(0.1,0.2$ and 0.3$)$. Release data showed that formulations containing highest amount of drug show fast and higher released rates than those formulations containing a small amount of drug. A prolong released was observed for the formulation containing lower amount of drug. In other words, with decreasing amount of drug in the matrix, it is noticed that the released rate becomes quite slower, this is due to the availability of more free void spaces through which lesser number of drug molecules will transport. Because drug release from the blend membranes is sustained by diffusion mechanism, the release rates are slow at lower amount of Flutamide. Similar release profiles were also reported by various researchers (Ritger et al., 1987).

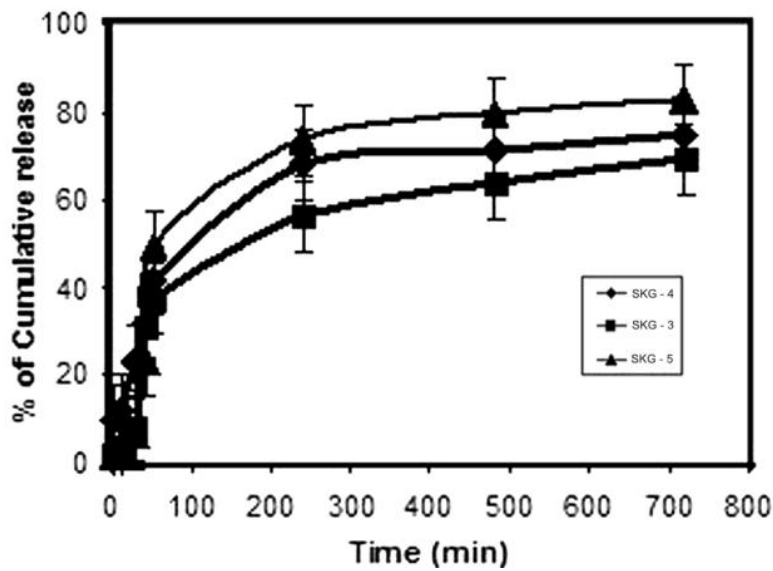

Fig. 6 (b): Percentage cumulative release of Flutamide through SA/KG blend hydrogel membranes containing different amounts of Flutamide: ( $\downarrow) 20 \%$ FLT, (घ) $10 \%$ FLT, and ( $\Delta$ ) $30 \%$ FLT at pH 7.4.

\section{Effect of cross-linking agent}

The percent of cumulative release data vs. time plots for blend membranes with varying amounts of GA (0.01, 0.05 and 0.1 ) at a fixed amount of drug $(0.1 \mathrm{~g})$, and at a fixed ratio of polymer blend membrane (0.9SA:0.1KG) are shown in Fig 6 (c). The $\%$ cumulative release is quite fast and large, at lower amount of GA, whereas the release is quit lower at higher amount of GA. Probably, at higher concentration of GA, polymeric chains become rigid due to the contraction of micro voids, thus decreasing $\%$ 
cumulative release of Flutamide through the polymeric matrices. As expected, the release becomes slower at higher amount of GA, but becomes faster at lower amount of GA.

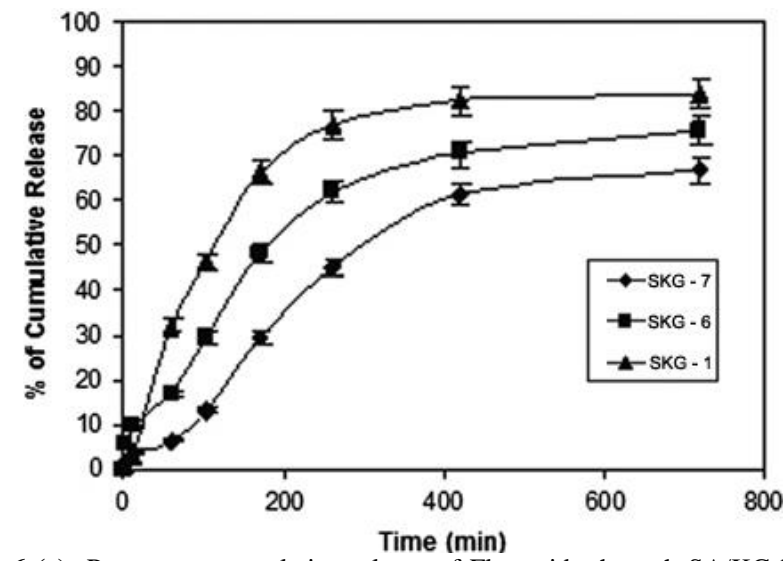

Fig. 6 (c): Percentage cumulative release of Flutamide through SA/KG blend hydrogel membranes containing different amount of crosslinker: ( $)$ 30\% GA, (-) $20 \% \mathrm{GA}$, and ( $\mathbf{\Delta}) 10 \% \mathrm{GA}$ at $\mathrm{pH} 7.4$.

\section{Drug release kinetics}

In order to establish a link between drug release and molecular transport parameters, we have fitted the release data to an empirical equation (Korsema., 1987):

$$
\left(M_{t} / M_{\infty}\right)=k t^{n} \ldots .3
$$

Where Mt / M $\alpha$ represents the fractional drug release at time $\mathrm{t}, \mathrm{K}$ is a constant characteristic of the drug polymer system and $n$ is an empirical parameter characterising the release mechanism. Using the least- squares procedure, we have estimated the values of $\mathrm{n}$ and $\mathrm{k}$ for all formulations using equation (3) and these results are presented in Table 2. If $n=0.5$, drug diffuses and release out of the polymer matrix following. fickian diffusion. For $\mathrm{n}>0.5$, anomalous are non fickian type drug diffusion occurs. If $n=1$, a completely non fickian or case II release kinetics is operative. The intermediary values ranging between 0.5 and 1.0 or attributed to anomalous type of diffusion transport (Ritger et al., 1987). The values of $\mathrm{k}$ and $\mathrm{n}$ as shown a dependence on the extent of crosslinking, the percentage of drug loading and KG content in the matrix. Values of $\mathrm{n}$ for membranes are calculated by varying the amount of $\mathrm{KG}(0.1,0.15,0.2 \mathrm{gms})$, keeping flutamide $(10 \%)$ and GA ( $2 \mathrm{ml})$ constant, and range from 0.700 to 0.862 , leading to a shift of anomalous type. The flutamide loaded membranes containing different amounts of flutamide and GA exhibited $n$ values ranging from 0.473 to 0.686 , and the values for different formulations are presented Table 2, indicating the shift transport of anomalous type release. This may be due to the reduction in the reasons of low micro viscosity and closure micro cavities in swollen state of the polymer. Similar findings were also observed by Lyu et al., (Lyu et al., 2005) from this drug release structures where they reported the effect of monomer, drug and crosslinker content on dissolution kinetics.

Table 2: Results of \% of release kinetics parameters (k, $\mathrm{n}$ and $\mathrm{r}$ ) of drug in different blend membrane formulations

\begin{tabular}{cccc}
\hline Sample code & k & n & r \\
\hline SKG-1 & 0.169 & 0.686 & 0.948 \\
SKG-2 & 0.069 & 0.478 & 0.943 \\
SKG-3 & 0.084 & 0.483 & 0.962 \\
SKG-4 & 0.126 & 0.536 & 0.950 \\
SKG-5 & 0.147 & 0.642 & 0.943 \\
SKG-6 & 0.128 & 0.535 & 0.971 \\
SKG-7 & 0.142 & 0.576 & 0.992 \\
\hline
\end{tabular}

\section{CONCLUSIONS}

The $\mathrm{SA} / \mathrm{KG}$ blend membranes were prepared by using KG with different concentrations of SA, drug and Glutaraldehyde. The concentration of KG and Glutaraldehyde had an influence on the morphology, mechanical, and swelling property of the blend membranes. The blend membranes exhibit a much higher stiffness than pure SA membrane, indicating that this strategy may allow for the use of SA-based structures in tissue engineering applications requiring some mechanical features. The $\%$ of swelling of the blend membranes increased with either increasing of SA content or decreasing crosslink densities and $\mathrm{KG}$ content. The maximum swelling of the membranes was achieved in about 10-12 h. The drug release profile was affected by the composition of the blend membranes. The drug release was fast at the initial period, in agreement to the water uptake behaviour; however, almost constant drug release was observed at the later time period. The blend membranes prepared with the lowest amounts of Karaya gum and cross-linking agent which showed the highest percentage of drug release. These preliminary results suggested that $\mathrm{SA} / \mathrm{KG}$ blend membranes could be suitable to release bioactive components for stimulating cell differentiation and proliferation or drugs, such as anti-inflammatory and antibiotics, to induce therapeutic effects in tissue engineering strategies.

\section{ACKNOWLEDGEMENTS}

This work was encouraged by the department of Polymer Science \& Technology, S. K. University, Ananthapuramu, Andhra Pradesh, India.

\section{Financial support and sponsorship: Nil.}

Conflict of Interests: There are no conflicts of interest.

\section{REFERENCES}

D. Sahoo, S. Sahoo, P. Mohanty, S. Sasmal, P.L. Nayak. Chitosan: a new versatile biopolymer for various applications. Designed Monomers and Polymers. 2009; 12, 377-404.

S. Sahoo, A. Sasmal, R. Nanda, A. R. Phani, P. L. Nayak. Synthesis of chitosan- poly caprolactone blend for control delivery of ofloxacin drug. Carbohydrate Polymers. 2010; 79, 106-113.

U. Edlund, A. C. Albertsson. Degradable polymer microspheres for controlled drug delivery. Advances in Polymer Science. 2002; 157, 67112. 
A. Jain Rajeev. The manufacturing techniques of various drug loaded biodegradable poly(lactide-co-glycolide) (PLGA) devices. Biomaterials.2002; 21, 2475-2490.

K.R. Kamath and K. Park. Biodegradablehydrogels in drug delivery, Advanced Drug Delivery Reviews.1993; 11, 59-84.

E. Fortunati, I. Armentano, Q. Zhou, A. Iannoni, E. Saino, L. Visai, L.A. Berglund, J. M.Kenny. Multifunctional bio-nanocomposite films of poly(lactic acid), cellulose nano-crystals and silver nano-particles. Carbohydrate Polymers. 2012; 87, 1596-1605.

H. Okuno, T. Uragami. Characterization of permeation and separation of aqueous alcohol solutions through cross-linked pullulan membranes. Polymer. 1992; 33, 1459-1469.

L. Zhang, D. Zhou, H. Wang, S. Cheng. Ion-exchange membranes from blends of cellulose cuoxam with alginate. Journal ofMembrane Science.1997; 124, 195-201.

A. Chanachi, R. Jiraratananon, D. Uttapap, G.Y. Moon, W.A Anderson, R.Y.M. Huang. Pervaporation with Chitosan/hydroxyl ethylcellulose (CS/HEC) blended membranes. Journal of Membrane Science. 2000; 166, 271-280.

S. Cao, Y. Shi, G. Chen. Influence of acetylation degree of cellulose acetate on pervaporation properties for $\mathrm{MeOH} / \mathrm{MTBE}$ mixture. Journal of Membrane Science. , 2000; 165: 89-97.

K.S. Soppimath, T.M. Aminabhavi, A.R. Kulkarni, W.E Rudzinsiki. Biodegradable polymeric nano-particles as drug delivery devices. Journal Controlled Release. 2001; 70, 1-20.

C. Gardner. Drug Targeting: Potentials and Limitations. In: Topics in Pharmaceutical Science. (Eds) D.D. Briemer, P. Speiser. 1983; 291.

411

G. Gregoriadis. Targeting of drugs. Nature. 1977; 265, 407-

G. Gregoriadis, J. Senior, G. Poste, (Eds). Targeting of Drugs with synthetic systems. NATO-yASI series (No. 113). Plenum Press, NY. 1977; 1986.

Y. Yuan, B.M.Chesunatt, G utturkar, W O Haggard, Y Yang, J L ONG and J D Bumgardner. The effect of crosslinking of chitosan microspheres with genipin on protein release. Carbohydrate polym. 2007; $68,56-567$

J Du, J Dai, J Liu, T dankovich. Novel PH Sensitive polyelectrolyte Carboxy methyl konjacglucomannan - chitosan beads as drug carriers. Funct. Polym. 2006; 66, 1055-1061

HCB Paula, FJS. Gomes, RCM Paula. Swelling studies chitosan / cashew nut gum physical gels. Carbohydra. Polym. 2002; 48, 313-318

A. Mochizuki, S. Amiya, Y. Sato, H. Ogawara, S. Yamashita. Pervaporation separation of water/ethanol mixtures through polysaccharide membranes. IV. The relationships between the permselectivity of alginic acid membrane and its solid state structure. Journal of Applied Polymer Science. 1977; 40, 385-400.
F.G. Fischer, H. Dorfel. Thepolyuranic acids of brown algae, Hoppe-Seyler'sZeitschrift fur PhysiologischeChemie. 1955; 302, 186.

Haung, B. Larean, O. Smolder. A study of construction of alginic acid by partial acid hydrolysis. ActaChemicaScandinavica, 1966; 20, 183-190.

S.T. Moe, K.I. Draget, G.S. Break, O. SmidsrodAlginates, in: A.M. Stephen (Ed.). Food polysaccharides and their applications. first ed., Marcel Dekker, New York, 1995; 245-286.

M N Prabhakar, U Sajan kumarji rao, P. Kumara babu, M C S Subha and K Chowdoji rao. Interpenetrating polymer network hydrogel membranes of PLA and SA for control release of Penicillamine drug. Indian Journal of advances in chemical science, 2013; 1 (4), 240-249.

Venkata Prasad C., Yerriswamy B., Mallikarjuna B., Sreekanth K.C., Subha M.C.S., Chowdoji Rao K.. Preparation and characterization of interpenetrating polymer network beads for controlled release of acebutolol hydrochloride. Advances in PolymerTechnology, 2012; 31, 8799.

R. W. Korsmeyer, S. R. Lustig, N. A. Peppas. Solute and penetrant diffusion in swellable polymers. I. Mathematical modeling. Journal of Polymer Science B Polymer Physics, 1986; 24(2), 395-408.

P. L. Ritger, N. A. Peppas. A simple equation for description of solute release ii. Fickian and anomalous release from swellable devices. Journal of Controlled Release, 1987; 5, 37-42.

S.P. Lyu, R. Sparer, C. Hobot, K. Dang. Adjusting drug diffusivity using miscible polymer blends. Journal of Controlled Release, $2005 ; 102,679-687$.

\section{How to cite this article:}

Nagarjuna G, Babu PK, Maruthi Y, Parandhama A, Madhavi C, Subha MCS, Chowdojirao K. Interpenetrating Polymer Network Hydrogel Membranes of Karayagum and Sodiumalginate for Control Release of Flutamide Drug. J App Pharm Sci, 2016; 6 (12): 011-019. 